INPLASY

PROTOCOL

To cite: Li et al. Effect of Liuweiwuling (LWWL) tablet on biochemical and virological parameters, and quality of life in patients with hepatitis $B$ virus-related cirrhosis: protocol for a systematic review and meta-analysis. Inplasy protocol 202080010. doi:

10.37766/inplasy2020.8.0010

Received: 04 August 2020

Published: 04 August 2020

Corresponding author:

Xiujing Liu

641098338@qq.com

Author Affiliation:

Liaocheng People's Hospital

Support: 2015ZRA15027

Review Stage at time of this submission: The review has not yet started.

Conflicts of interest: The authors declare that they have no competing interests.

\section{Effect of Liuweiwuling (LWWL) tablet on biochemical and virological parameters, and quality of life in patients with hepatitis $B$ virus-related cirrhosis: protocol for a systematic review and meta-analysis}

\author{
Li, D1; Zhu, M2; Zhou, CH³; Liu, XJ4.
}

Review question / Objective: Is Liuweiwuling (LWWL) tablet effective on biochemical and virological parameters, and QoL in patients with hepatitis B virus-related cirrhosis (HBVC)? Condition being studied: Liuweiwuling tablet, biochemical and virological parameters, quality of life and hepatitis B virusrelated cirrhosis.

Information sources: Electronic databases including PubMed, Medline, Cochrane Library, Google Scholar, Web of Science (WOS), Excerpt Medica Database (Embase), China National Knowledge Infrastructure (CNKI), Chinese Biomedical Literature Database (CBM), China Scientific Journal Database (CSJ) and Wanfang Database will be systematically searched for eligible clinical trials from January 2000 to August 2020. Language is limited with English and Chinese.

INPLASY registration number: This protocol was registered with the International Platform of Registered Systematic Review and Meta-Analysis Protocols (INPLASY) on 04 August 2020 and was last updated on 04 August 2020 (registration number INPLASY202080010).

\section{INTRODUCTION}

Review question / Objective: Is Liuweiwuling (LWWL) tablet effective on biochemical and virological parameters, and QoL in patients with hepatitis B virusrelated cirrhosis (HBVC)?
Rationale: Liuweiwuling (LWWL) tablet, a kind of plant-derived traditional Chinese medicine preparation, has been widely applied as a promising adjunctive drug for hepatitis B virus-related cirrhosis (HBVC). However, its exact clinical efficacy and 
safety is still not well investigated. In this study, we aimed to summarize the efficacy of LWWL tablet on biochemical and virological parameters, and quality of life (QoL) in patients with HBVC through the meta-analysis, in order to.

Condition being studied: Liuweiwuling tablet, biochemical and virological parameters, quality of life and hepatitis B virus-related cirrhosis.

\section{METHODS}

Search strategy: To perform a comprehensive and focused search, experienced systematic review researchers will be invited to develop a search strategy. The plan searched terms are as follows: "hepatitis B virus" or "liver cirrhosis" or "hepatitis B cirrhosis" or "hepatitis B virusrelated cirrhosis" or "HBV-cirrhosis" or "HBV-C" combined with "Liuweiwuling" or "Liuweiwuling tablet" or "LWWL" et al. An example of search strategy for PubMed database shown in Table 1 will be modified and used for the other databases.

Participant or population: Patients were diagnosed hepatic cirrhosis that caused by HBV. No restrictions regarding age, gender, racial, region, education and economic status in this analysis. Patients who had no hepatocellular carcinoma or other malignant tumor.

Intervention: HBVC patients in the experimental group must be treated with conventional treatment (including routine liver protection therapy or oral antiviral therapy,) combined with LWWL tablet.

Comparator: In the control group, HBVC patient treated with the same conventional treatment as experimental group.

Study designs to be included: All available comparative clinical trials that assessed the efficacy of LWWL tablet in the treatment of HBVC patients will be included.

Eligibility criteria: This study will include randomized controlled trials (RCTs) or
quasi-RCTs, and high-quality prospective cohort studies that investigated the efficacy of LWWL tablet on biochemical and virological parameters, and QoL in patients diagnosed with HBVC will be included in this systematic review. Papers without sufficient available data, non-peer reviewed studies, non-comparative clinical trials, literature reviews, meta-analysis, meeting abstracts, case reports, commentaries, letter to the editor, and other unrelated researches will be excluded from analysis.

Information sources: Electronic databases including PubMed, Medline, Cochrane Library, Google Scholar, Web of Science (WOS), Excerpt Medica Database (Embase), China National Knowledge Infrastructure (CNKI), Chinese Biomedical Literature Database (CBM), China Scientific Journal Database (CSJ) and Wanfang Database will be systematically searched for eligible clinical trials from January 2000 to August 2020. Language is limited with English and Chinese.

Main outcome(s): The primary outcomes will include: I) Liver function indexes: Liver function of patients with HBVC was assessed in terms of total bilirubin (TBIL), serum albumin (ALB), ALT and AST levels; II) Virological indicators: HBV DNA levels, HBeAg status and seroconversion; III) QoL as evaluated by Karnofsky score.

Additional outcome(s): Secondary outcomes will include: I) Model for endstage liver disease (MELD) and Child-Pugh score. II) Alpha-fetoprotein (AFP) level; III) Liver fibrosis indexes: hyaluronic acid (HA), laminin (LN), type III procollagen (PC III) and type IV collagen (CIV) levels. IV) Immune function indicators: CD3+, CD4+, CD8+, NK cells percentage, and CD4+/ CD8+ cell ratios; V) Adverse events: toxicity was graded from 0 to IV in severity on the basis of the World Health Organization (WHO) recommendations.

Data management: Two investigators (Li D and Zhu M) will be responsible for the data extraction independently according to the Cochrane Handbook for Systematic 
Reviews of Intervention. The following data will be extracted from eligible literatures: I) Study characteristics and methodology: country of study, the first author, year of publication, study design, sample size, periods of data collection, total duration of study and follow-up duration, et al. II) Participant characteristics: age, gender, ethnicity, inclusion and exclusion criteria, et al. III) Interventions: therapeutic means, manufacturer of the drugs, dosage of LWWL tablet, administration route and cycles, duration of treatment and follow-up time, et al. IV) Outcome and other data: (a) Liver function indexes (TBIL, ALB, ALT and AST); (b) Virological indicators (HBV DNA levels and $\mathrm{HBeAg}$ status); (c) Liver fibrosis indexes (HA, LN, PC III and CIV levels); (d) Immune indicators: (CD3+, CD4+, CD8+, NK cells percentage, and CD4+/CD8+ cell ratios); (e) MELD and Child-Pugh score; (f) Other data (QoL, AFP and adverse effects, et al.). Dealing with missing data: we will attempt to contact the authors to request the missing or incomplete data. If those relevant data are not acquired, they will be excluded from the analysis.

Quality assessment / Risk of bias analysis: Two review authors (Li D and Zhu M) will independently assess the methodological quality of the included trials by using the following criteria described in the Cochrane Handbook for Systematic Reviews of Interventions: random sequence generation, allocation concealment, blinding of participants and personnel, blinding of outcome assessment, incomplete outcome data, selective reporting and other bias. Evidence quality will be classified as low risk, high risk, or unclear risk of bias. EPOC guidelines will be used to assess the risks of non-RCTs. Any disagreements will be resolved via discussion with a third researcher (Zhou CH).

Strategy of data synthesis: Review Manager 5.3 (Nordic Cochran Centre, Copenhagen, Denmark) and Stata 14.0 (Stata Corp., College Station, TX, USA) statistical software will be used to pool the data and carry out the data analysis. Heterogeneity between studies will be assessed using the Cochran's $Q$ and Higgins 12 statistic. $P<0.1$ or $I 2>50 \%$ will be considered as showing considerable heterogeneity, and the random effect model will be used. Otherwise, a fixed effect model will be used for data analysis. Dichotomous data will be recorded as risk ratio (RR) with $95 \%$ confidence intervals (CIs). Continuous data will be presented as standardized mean difference (SMD) with their Cls. A two-tailed $P<0.05$ was considered statistically significant.

Subgroup analysis: Subgroup and metaregression analysis based on cirrhosis stage, treatment period, dose of LWWL tablet, level of risk of bias, and other unpredictable factors will be performed to detect the source of heterogeneity.

Sensibility analysis: Sensitivity analysis will be carried out to assess the reliability and robustness of the pooled results via eliminating trials with low quality. A summary table will report the results of the sensitivity analyses.

Language: Language is limited with English and Chinese.

\section{Country(ies) involved: China.}

Other relevant information: a) Publication bias analysis Funnel plot, Begg's and Egger regression test will be performed to analyze the existence of publication bias if 10 or more studies are included in this meta-analysis. If reporting bias is suspected, we will consult the study author to get more information. If publication bias existed, a trim-and-fill method should be applied to coordinate the estimates from unpublished studies, and the adjusted results were compared with the original pooled RR. b) Evidence evaluation The Grading of Recommendations, Assessment, Development, and Evaluation (GRADE) system will be used for assessing the strength of the body of evidence. According to the grading system, the quality of evidence will be evaluated as high, moderate, low, and very low level. 
Keywords: Xliuweiwuling tablet; hepatitis B virus-related cirrhosis; efficacy; metaanalysis.

Dissemination plans: We will disseminate the results of this systematic review by publishing the manuscript in a peerreviewed journal.

Contributions of each author:

Author 1 - Ding Li - Conceptualization, Data curation, Formal analysis, Investigation, Methodology, Resources, Software, Supervision, Visualization, Writing-original draft.

Author 2 - Min Zhu - Data curation, Formal analysis, Investigation, Methodology, Visualization, Writing-original draft.

Author 3 - Changhui Zhou - Funding acquisition, Investigation, Methodology, Validation, Writing-original draft, Writingreview \& editing.

Author 4 - Xiujing Liu - Conceptualization, Project administration, Resources, Software, Supervision, Validation, Writingreview \& editing. 